\title{
The Novel Concept of Single Path Multiple Access (SPMA) Using New Advanced Antenna Technology
}

\author{
Muhammad Usman Sheikh, Student Member, IEEE, and Jukka Lempiäinen, Senior Member, IEEE \\ Department of Communications Engineering, Tampere University of Technology \\ P.O. Box 553, FIN-33101, Tampere, Finland \\ muhammad.sheikhetut.fi, jukka.lempiainenetut.fi
}

\begin{abstract}
The aim of this paper is to introduce a novel frequency reuse concept especially for macro cellular networks to substantially increase the mobile network capacity, and simultaneously to avoid the implementation of low efficient small cells. Single path multiple access (SPMA) utilizes the characteristics of independent propagation paths for particular geographical location in the coverage area of mobile network. When communication between base station and mobile station happens through only one independent propagation path, frequency resources can be reused in $5 \mathrm{~m} \times 5 \mathrm{~m}$ areas, or even more often in $1 \mathrm{~m} \times 1 \mathrm{~m}$ areas. Thus, the capacity of the network will be increased dramatically, and it can be managed in centralized manner at certain macro site locations. In already deployed cellular networks, these macro sites are mostly easily available, and that would help to implement SPMA to enhance the network capacity.
\end{abstract}

Simulation results provided in this paper show the applicability of single path multiple access technique, by limiting the radiation of signal as single path propagation between base station and mobile station.

Index Terms - System Capacity, Mobile Networks, Radio Access, Multipath Propagation

\section{INTRODUCTION TO MOBILE NETWORK LAYOUTS}

NEW capacity requirements for data traffic in mobile networks and the requirement of high practical data rates in cellular networks have finally started discussions about possible network layouts in the future. It has been noted several times that traditional macro cells are not able to offer equal data rates for the users over the entire network, and most of the network capacity has been lost because of the interference coming from neighbor cells and sites. Also new network layers as micro cells, small cells, and femto cells have been proposed to improve system capacity in urban high density traffic areas [1].

However, one of the key strategic goals in the deployment of mobile networks is to avoid the addition of new sites and cells because of increased fixed costs such as electricity, transmission, rentals, etc. Thus, macro cells are of high importance for mobile operators' point of view, and their efficient utilization should be guaranteed. Moreover, smaller cells should be used only whenever no other choice is available, and even then maybe together with macro cells. Addition of small cells not only adds the CAPEX rather it also increases OPEX for mobile operators. To avoid deployment of small cells, advanced antenna solutions are considered as an alternate choice for the mobile operators [2]. The research of these advanced antenna solutions includes high order sectoring [3-4], employing multiple fixed beam antennas and adaptive/smart antenna approach [5-7], or even massive MIMO approach [8-9]. These studies show that macro cell capacity can be increased by $100-200 \%$ depending on system layout and used technologies [2].

This capacity improvement helps for some years but it is still needed an advanced and a complete long-term solution to avoid extensive utilization of small cells. Hence, a totally new radio resource reuse approach is needed for macro cells to substantially increase the network capacity for the future use. All today's radio access concepts are more or less based on "cellular thinking" where one cell (macro, micro, small, pico, or femto) offers a certain coverage area, and thus also causes interference in unwanted directions. Adaptive antenna based Spatial Division Multiple Access (SDMA) is slightly different but still based on a certain antenna pattern of 5-10 degrees.

In order to keep the macro cell layout, and to increase radically frequency reuse, "a giant leap" is needed at concept level of radio access. This leap could be related to new antenna materials, and to new opportunities to create a communication between base station and mobile station only through one multipath instead of several multipath components. This approach is also changing a traditional "cellular approach" at least partially.

\section{Propagation Channel and Multipath Components}

Fig.1a shows the non-line-of-sight (NLOS) scenario between macro base station (TX) and three mobile station locations A, $\mathrm{B}$, and $\mathrm{C}$ at a distance of $5 \mathrm{~m}$ from each other in $2 \mathrm{D}$ environment. Both base station and mobile stations can transmit $(\mathrm{T})$, or receive $(\mathrm{R})$ the signal. The radio propagation channel between transmitting and receiving end is reciprocal, and thus radio signal propagates via similar multipaths to both directions. Multipath propagation is possible due to three propagation mechanisms: reflection, diffraction, and scattering. Base station antenna height is set clearly above the average rooftops of the buildings to have wider coverage area, and the mobile stations are at the street level with the small separation of five meter from each other.

Ray tracing techniques have been extensively employed as a theoretical prediction tool, and for the characterization of the radio propagation environment. Determination of multipath components between the Transmitter (TX) and Receiver (RX) is the first step towards the computation of received electric field or power at the receiver. By using ray tracing techniques based on Shoot and Bouncing Ray (SBR), or Image Theory (IT) algorithm, all possible multipath components can theoretically be found between each TX/RX locations [10-12]. 


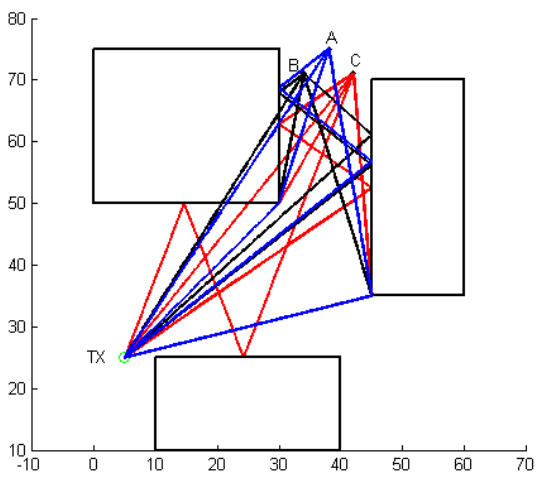

(a)

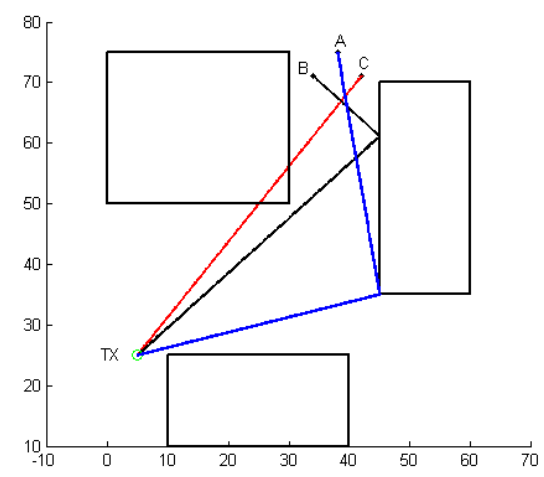

(b)

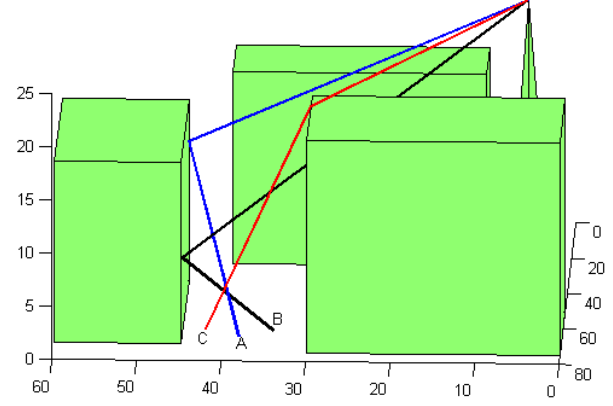

(c)

Fig.1(a) Multipath propagation in NLOS environment between base station, and three mobile locations A, B and C at different locations in 2D environment. Fig.1(b) Single path propagation in 2D environment. Fig.1(c) Single path propagation in 3D environment.

Fig.1a shows different multipath rays arriving at three different mobile locations from base station in 2D environment. All presented multipath components with finite reflections and diffraction shown in Fig.1a were found by using Image Theory based ray tracing algorithm [11]. Received signal at each mobile location is the sum of attenuated, slightly time delayed, and phase shifted replicas of the transmitted signal. In Fig.1b, only single multipath component arriving at three different mobile locations is shown. Now, each multipath component has different path and arrives at each receiver location from different direction. Finally, Fig.1c shows single path propagation between base station and three different mobile locations at a distance of $5 \mathrm{~m}$ from each other in 3D environment from different angle. Wall reflected, wall diffracted, and rooftop diffracted signal paths are visible in Fig.1c.

To have a better view of radio wave propagation in real world, 2D ray path needs to be converted into 3D ray paths. The propagation paths in $2 \mathrm{D}$ plane can be visualized in $3 \mathrm{D}$ plane by the approach defined in [10-12], and as shown in Fig.2. It was found that two 3D paths are possible i.e. with and without ground reflection for each 2D ray. Ground reflection point $(\mathrm{G})$ can be found by using the image of transmitter with respect to ground plane, or by using the information of height of transmitter $\left(H_{T}\right)$, height of receiver $\left(H_{R}\right)$, and the distance (d) between them. Ground reflected ray follows the law of reflection, and the angle of incidence $\left(\theta_{i}\right)$ of incident ray equals to the angle of reflection $\left(\theta_{r}\right)$ of reflected ray as shown in Fig.2, assuming a flat ground. Later, radio propagation loss for 3D rays can be calculated by using free space path loss along with the information of number of reflections and diffractions.

Moreover, each multipath component is characterized by its power, time delay at arrival, Direction of Departure (DoD), Angle of Departure (AoD), Direction of Arrival (DoA), and Angle of Arrival (AoA). The angle in horizontal plane (azimuth plane) between base station and mobile station with respect to base station defines $\mathrm{DoD}$, and with respect to mobile station defines DoA. Similarly, the angle in vertical plane (inclination angle) between base station and mobile station with respect to base station defines $\mathrm{AoD}$, and with respect to mobile station defines AoA. The received power at the receiver is the sum of all multipath components added coherently (in phase addition).

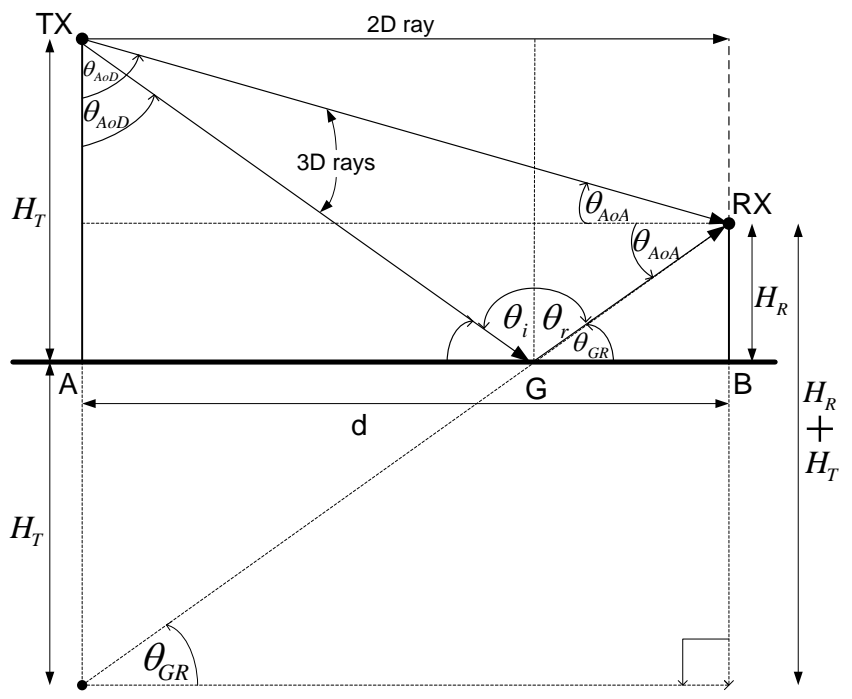

Fig.2. Conversion of $2 \mathrm{D}$ rays into $3 \mathrm{D}$ rays.

To have more in depth analysis of signal propagation for mobile stations located very close to each other, consider two mobile stations i.e. MS1 and MS2 separated by just $1 \mathrm{~m}$ distance in a NLOS environment with BS (TX) as shown in Fig.3a. Multipath components shown in Fig.3b are based on ray tracing with maximum number of four reflections, two diffractions, single rooftop diffraction, and single ground reflection. Fig.3c shows all possible multipath components in $3 \mathrm{D}$ environment.

Fig.4 shows the amplitude and delay profile of available multipath components arriving at two different mobile stations separated by $1 \mathrm{~m}$ as shown in Fig.3a. Due to different time delay for each multipath component arriving at receiver location, the received signal is dispersed in time domain. It can be noted that for individual mobile station, some multipath components are arriving at almost the same time, or with a small time difference, even though they followed different paths. As receivers, MS1 and MS2, are located close to each 


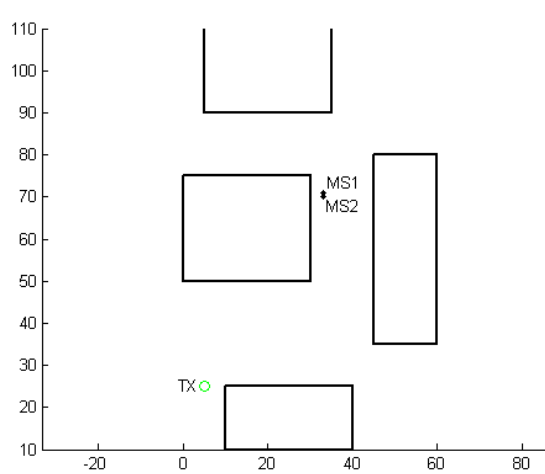

(a)

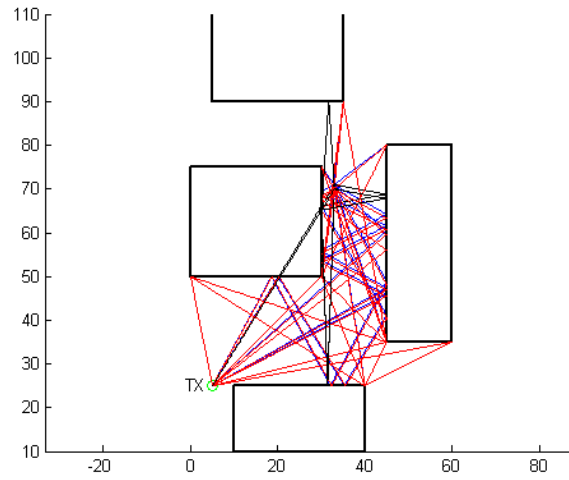

(b)

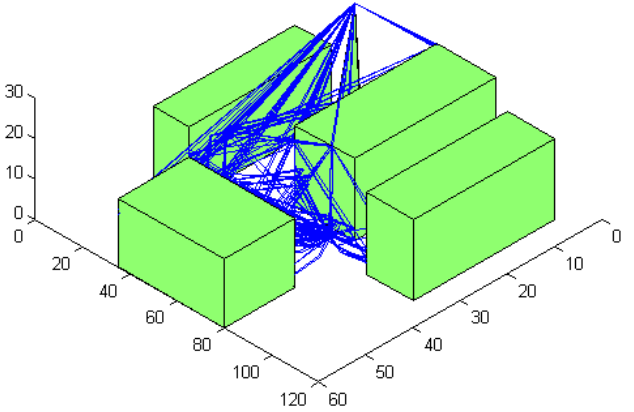

(c)

Fig.3(a) NLOS radio propagation channel between base station, and two mobile stations MS1 and MS2 at two different locations. Fig.3(b) Multipath propagation in 2D environment. Fig.3(c) Multipath propagation in 3D environment.

other, they have almost identical power and delay profile. It can be seen that for both mobiles, only few multipath components with a short time delay constitute a major contribution to the total received power. It means multi- path components arriving after certain delay can be neglected.

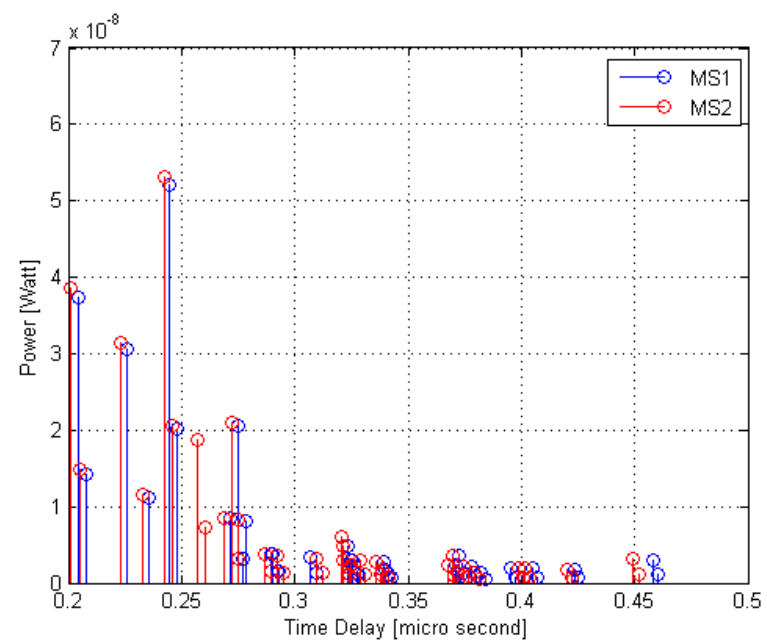

Fig.4. Multipath components of MS1 and MS2 with different time delays.

Fig.5 presents even more detailed analysis of arrived multipath components. Fig. 5 highlights the DoD of only 10 strongest multipath components of both mobile stations that received at different time instants. It can be seen that multipath components for MS1 and MS2 are mainly radiated from transmitter in 3-5 main directions in azimuth plane, where reflections and diffractions occur. Altogether there were 42 and 46 multipath components for MS1 and MS2, respectively, but 10 strongest components represent $77.79 \%$ and $73.8 \%$ of the total received power for MS1 and MS2, respectively. Now, only one strong multipath, not necessarily the strongest one, can be used to establish a valid communication link between base station and mobile station, if antennae at both ends are able to point the radiation precisely in correct direction. The strongest multipath component for MS1 and MS2 shown in Fig.5 contributes $19.53 \%$ and $17.30 \%$ of the total received power, respectively, which is around the typical value in nonline-of-sight (NLOS) environment.

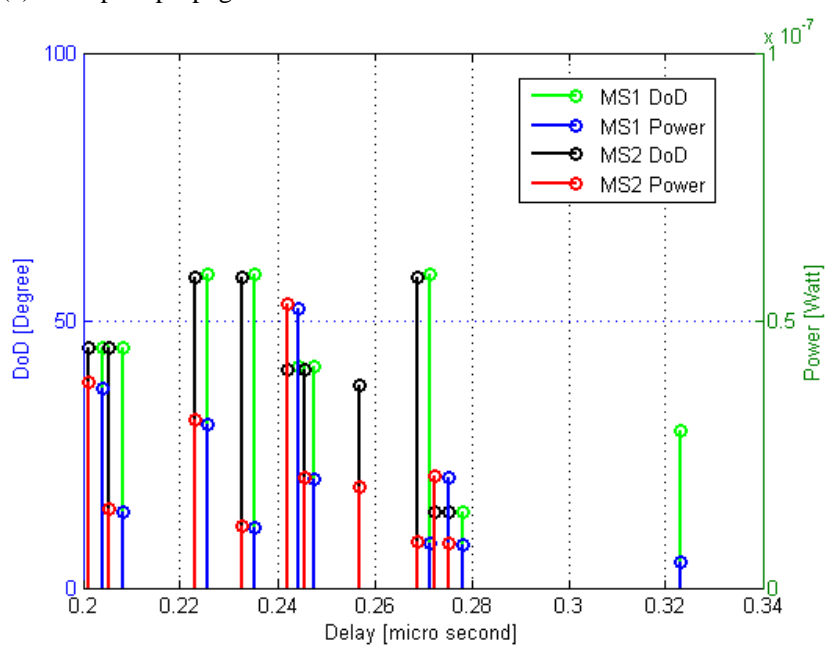

Fig.5.Amplitude and DoD of 10 strongest multipath components for MS1 and MS2 against time delay.

This amount of received power is enough for communication between transmitter and receiver. If this main component is destroyed, or corrupted by any means, then the second or the third best component can be used. Second strongest multipath component represents $14.02 \%$ and $12.57 \%$ of the total received power; similarly third strongest component adds $11.47 \%$ and $10.22 \%$ to total received power at MS1 and MS2, respectively.

\section{SINGLE PATH MULTIPLE ACCESS CONCEPT}

For each geographical location, received power is the sum of independent multipath components between base station and mobile stations. Thus, a single independent multipath component can be used to represent a single BS - MS connection, sharing the radio resources or utilizing the whole available frequency spectrum at each different location. Moreover, same radio and frequency resources can be reused, after every meter or few meters. If there are several mobile users sharing the frequency band, then it corresponds to multiple access, and thus radio access will be known as Single Path Multiple Access (SPMA). When frequency resources can be reused at every meter distance, available frequency spectrum can be reused 1,000,000 times per square kilometer, enhancing a huge network capacity. 


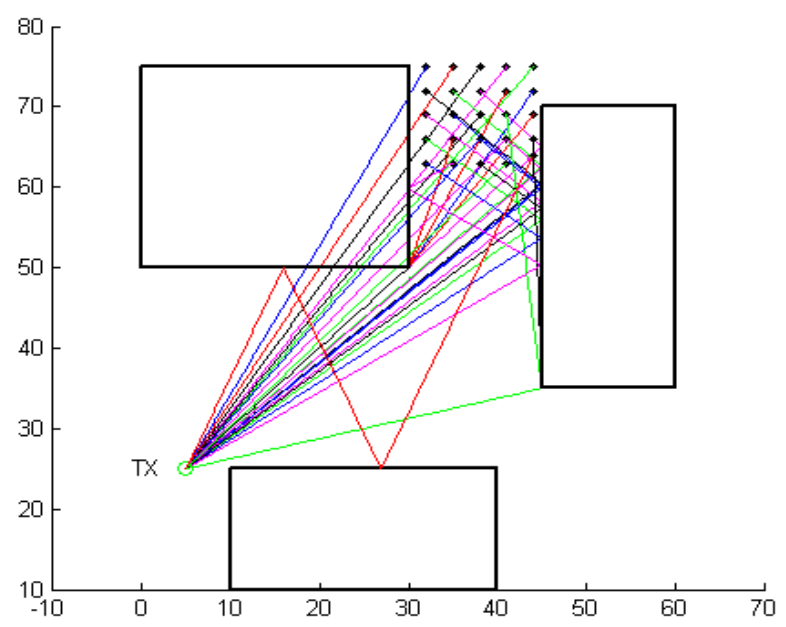

Fig.6. Single and independent path between BS (TX) and 25 mobile locations separated by $2 \mathrm{~m}$.

Fig.6 shows an example of reusing the frequency resources after every $2 \mathrm{~m} \times 2 \mathrm{~m}$ area by 25 different mobile stations, by establishing a radio connection between transmitter (TX) and receiver via single path. In order to have only single path connection to a certain location, radio propagation and thus the antenna radiation needs to be limited in a significantly narrow beam in an azimuth as well as in vertical plane. This limited radiation and propagation can be produced at both base station and mobile station ends, but especially at base station antenna.

Base station antenna is typically implemented at rooftops in macro sites, and thus it can have bigger size like $0.4 \mathrm{~m} \times 1.4 \mathrm{~m}$. In order to have narrow radiation pattern in azimuth or elevation directions, an antenna array is needed. Linear antenna array is an array of $\mathrm{N}$ antenna elements located at equal distance from each other in the same direction. Similarly, in two dimensional array, antenna elements are uniformly placed in $\mathrm{x}-\mathrm{y}$ plane with uniform spacing between them. Array Factor (AF) for one dimensional and two dimensional antenna arrays is given by equation (1) and (2), respectively. [7]

$$
\begin{gathered}
A F_{1 D}=\sum_{n=1}^{N} w_{n} \mathrm{e}^{-\mathrm{j} \frac{2 \pi}{\lambda} Z_{\mathrm{n}} \cos (\theta)} \\
A F_{2 D}=\sum_{n=1}^{N} w_{n} \mathrm{e}^{-\mathrm{j} \frac{2 \pi}{\lambda}\left(\mathrm{X}_{\mathrm{n}} \sin (\theta) \cos (\varphi)+\mathrm{Y}_{\mathrm{n}} \sin (\theta) \sin (\varphi)\right)}
\end{gathered}
$$

where in equation (1), $N$ is the number of elements, $w_{n}$ is the weighting factor, $\mathrm{Z}_{\mathrm{n}}$ is the spacing of $n^{\text {th }}$ element, and $\theta$ is the angle of elevation. In equation (2), $X_{n}$ and $Y_{n}$ are the spacing of antenna elements in $\mathrm{x}$ and y plane, respectively, and $\varphi$ is the angle of azimuth. 2D array can filter signal based on their angle of elevation and azimuth [7]. Array factor of linear one dimension array for different number of antenna elements with uniform spacing of $3 / 4 \lambda$ and weighting factor $w_{n}$ equal to one is shown in Fig.7. It can be seen in Fig.7, that magnitude of main lobe along with side lobe increases and beamwidth of antenna decreases by increasing the number of antenna elements, assuming equal weight factor.

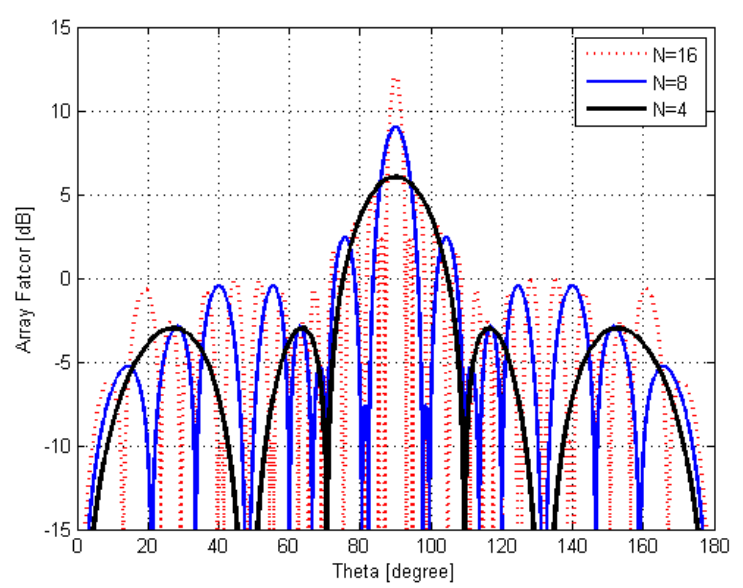

Fig.7.Magnitude of Array Factor (AF) for different number of antenna elements with equal spacing of $3 / 4 \lambda$.

Computational weighting factors can be used to obtain a desired pattern. The array of 8,16 and 32 elements provides $5.84^{\circ}, 2.46^{\circ}$ and $1.08^{\circ}$ of beamwidth, respectively. Therefore, a very large array of antenna is needed to achieve, for example, a half power beamwidth of $0.3^{\circ}$. This type of antenna array is most probably too big in practice, except if new materials and technologies like nanotechnology, or grapheme is used, which can squeeze the structure radically [13-16]. Hence, to achieve an extremely narrow beam, either antenna arrays with small elements spacing is needed as mentioned above, or a novel antenna concept is required.

Moreover, a key assumption for SPMA concept is based on expectation that new electrical materials (meta-materials, graphene, etc. [13-14]) will be used for antenna manufacturing. Graphene has recently gained much popularity and has got enormous attraction by the research industry due its exceptional electrical, mechanical, thermal and chemical properties [16]. Potential antenna and RF applications of graphene at microwave and terahertz frequencies are proposed by the researchers at [15]. It gives radically new possibilities to have narrow radiation pattern using graphene based antenna at nano scale level. It will not only reduce the size of antenna rather will also be able to serve the purpose of SPMA concept by employing narrow beams like "needle".

Conventional wide beam antenna covering the cell area is shown in Fig.8a. New antenna concept may mean, for example, a normal antenna covered by a new electrical material as shown in Fig.8b. This new electrical material should enable antenna to radiate in a certain direction of interest, and prevent radiation in other unwanted directions. Single and independent communication path for each user can be seen in Fig.8b. Simultaneously, this new material can be controlled at centralized macro base station. Antenna itself can be consisted of one element or several elements. This new antenna concept also takes into account the end-users' health. In new antenna concept, radio power is not focused in narrow direction to get high intensity as in case of antenna arrays. Thus, new antenna concept is much safer especially at mobile station end which is more critical for mobile users. 


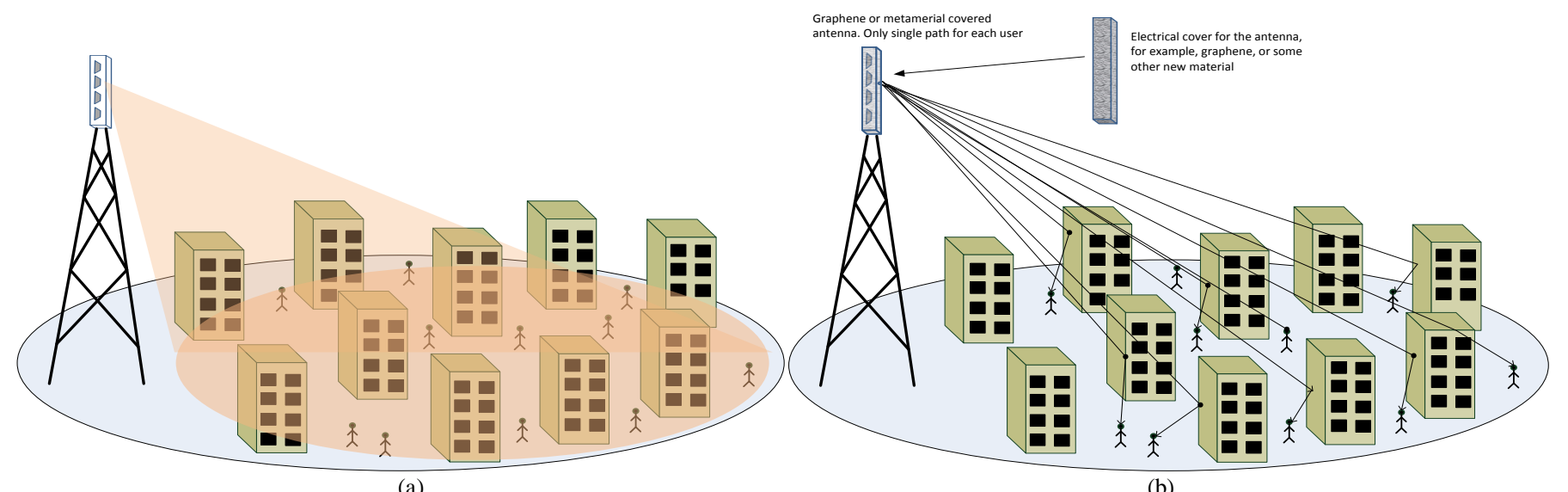

(a)

(b)

Fig.8(a). Conventional wide beam antenna concept. Fig.8(b). New type of antenna concept: a normal antenna surrounded by a new electrical material.

\section{SIMULATION RESULTS FOR ANTENNA REQUIREMENT}

For simulation purpose of this research work, MATLAB was used as a simulation tool for implementing a 3D ray tracer based on Image Theory (IT) algorithm. Unlike a quasi three dimensional environment, transmitter height was set above the height of buildings so that diffraction from the rooftops can be supported. Simulations with ray tracing were performed in 3D environment to show the preliminary requirements of the new proposed antenna concept, and to highlight the requirements of extremely narrow directed beam to avoid unwanted multipath components. All available multipath components with finite number of reflections and diffractions were studied, and analysis was made to select only single strong path, not necessarily the strongest one but an appropriate multipath in the direction of interest.

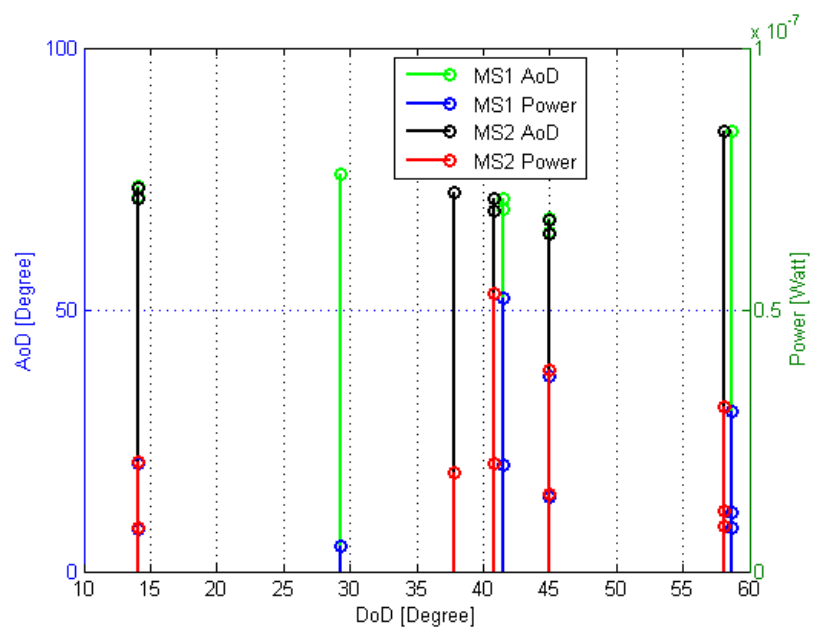

Fig.9.Amplitude and AoD of 10 strongest multipath components of MS1 and MS2 against DoD.

In order to show the degree of independence between two geographical locations $1 \mathrm{~m}$ apart from each other, the power, $\mathrm{DoD}, \mathrm{AOD}$, and AoA of received multipath components were analyzed. Assuming base station as a transmitting end and mobile station as a receiving end, Fig.9 shows the amplitude and angle of departure (AoD) of 10 strongest multipath components of MS1 and MS2 against the direction of departure (DoD), at transmitting end. For each geographical location, AoD along with DoD of interested multipath components defines the narrow beamwidth of the transmitting antenna for each particular user. Fig. 9 shows that the strongest multipath component for MS1 has $41.5^{\circ}$ of DoD with $71.3^{\circ}$ of AoD, whereas for MS2 the strongest multipath has $40.87^{\circ}$ and $71.13^{\circ}$ of DoD and AoD, respectively. Strongest multipath components for MS1 and MS2 were separated by $0.63^{\circ}$ and $0.17^{\circ}$ in the horizontal and vertical plane, respectively. The results presented in Fig.9 for MS1show that at the base station with respect to strongest multipath component $\left(41.5^{\circ}\right.$ of DoD), the closest and second closest unwanted multipaths are radiated at $45^{\circ}$ and $29.30^{\circ}$, respectively. For MS2 with respect to strongest multipath component $\left(40.87^{\circ}\right.$ of DoD), the closest and second closest unwanted multipaths are radiated at $37.81^{\circ}$ and $45^{\circ}$, respectively.

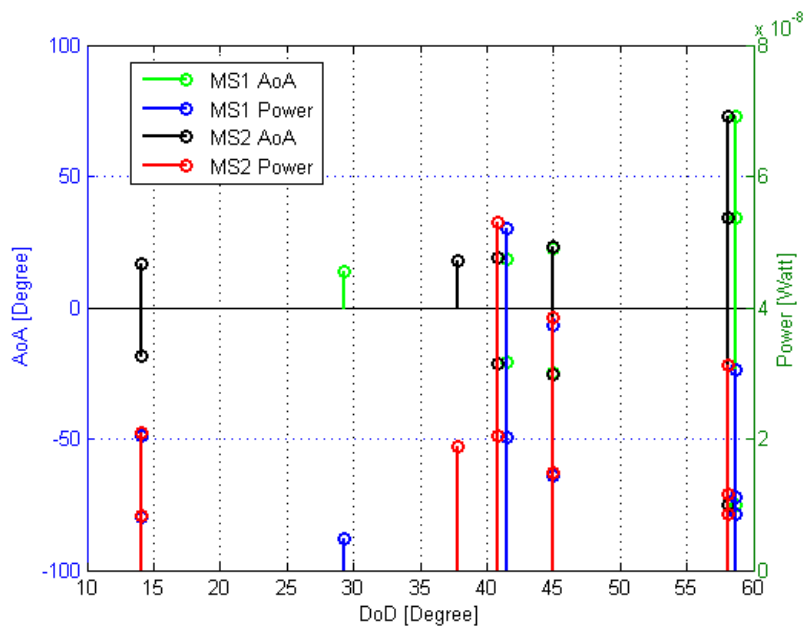

Fig.10.Amplitude and AoA of 10 strongest multipath components of MS1 and MS2 against DoD.

Fig.10 shows the amplitude and angle of arrival (AoA) of 10 strongest multipath components of MS1 and MS2 against the direction of departure (DoD), at receiving end. For each multipath component at any DoD, there always exist two AoA values; positive value representing free space signal without hitting the ground, and negative value representing ground reflected path. In Fig.10, only 10 strongest multipath components are shown, therefore at some DoD instants AoA with negative values are missing. If selected multipath for two mobile stations has very small separation in azimuth plane, then a free space path can be selected for one mobile station and ground reflected path for other mobile station to keep two multipaths independent from each other. 
Fig.11 shows the amplitude and angle of arrival of 10 strongest multipath components at the mobile station end (receiving end). At the receiving end, the strongest signal for MS1 and MS2 has $18.70^{\circ}$ and $18.87^{\circ}$ of AoA, respectively, separated by $0.17^{\circ}$ as shown in Fig.11. The negative values of angle of arrival shown in Fig.11 represent ground reflected signal paths. Second strongest multipath component was due to diffraction from the wall corner, and therefore having same DoD for both MS1 and MS2 with different AoD. But, the third strongest multipath component has $58.67^{\circ}$ and $58.11^{\circ}$ of DoD, and $84.06^{\circ}$ and $83.96^{\circ}$ of AoD for MS1 and MS2, respectively. Thus, we can say that $0.56^{\circ}$ and $0.1^{\circ}$ separation is needed at the base station in azimuth and vertical plane to avoid unwanted multipath components at the receiver with respect to third strongest multipath. Hence, if the strongest multipath component gets corrupted then the third strongest multipath component can be used instead of the second strongest due to wider separation in $\mathrm{DoD}$ and $\mathrm{AoD}$ compared to second strongest path.

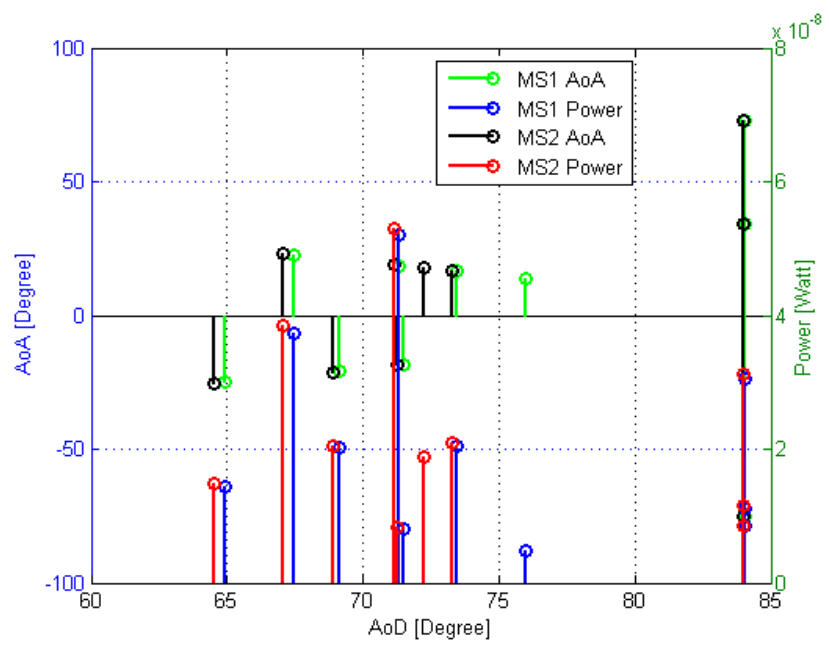

Fig.11. Amplitude and AoA of 10 strongest multipath components of MS1 and MS2 against AoD.

\section{CONCLUSIONS AND DISCUSSION}

In this paper, a novel concept of single path multiple access (SPMA) was introduced for gaining a multifold increase in capacity of centralized macro site in cellular networks. SPMA concept could be used also for fixed radio links (microwave links) for transmission between base stations and other network elements. Single path multiple access is based on an assumption that radiation from/to base station antennas can be directed to a very narrow target in a way that only single path propagation component between transmitter and receiver is used. This approach and concept would mean a huge increase in frequency reuse at centralized macro site locations but also bring a revolutionary change in typical cellular concept thinking.

The results obtained by $3 \mathrm{D}$ ray tracing simulations showed the requirement of antenna pattern to enable only very narrow directed beam. Based on propagation simulations, around $0.55^{\circ}-0.65^{\circ}$ separation is needed in azimuth plane between two independent multipaths for two users at geographical locations $1 \mathrm{~m}$ apart from each other. Similarly, $0.1^{\circ}-0.17^{\circ}$ separation is needed in vertical plane.
In the future work, first new possibilities should be studied to create very narrow radiation patterns (app. $0.1^{\circ}-0.2^{\circ}$ ) by using new materials i.e. nanotechnology, or by using traditional array approach. An alternate approach is to create non-traditional antenna design i.e. utilize possibilities to manage radiation such that only one specific direction radiates at time, and other directions can electrically be prevented. This non-traditional approach is also suitable for mobile terminal end because unhealthy intensity of radiation can be avoided, unlike in case of several elements antenna arrays.

\section{ACKNOWLEDGMENT}

Authors would like to thank European Communications Engineering (ECE) Ltd for funding this research work.

\section{REFERENCES}

[1] L. C. Wang, K. Chawla, L. J. Greenstein, "Performance Studies of Narrow-Beam Trisector Cellular Systems," International Journal of Wireless Information Networks, Vol. 5, No. 2, 1998, pp. 89-102.

[2] I.V. Stevanović, A. Skrivervik, J. R. Mosig, "Smart antenna systems for mobile communications". Laboratoire d'Electromagnetisme et d'Acoustique Ecole Polytechnique Federale de Lausanne, January 2003.

[3] J. Niemela, J. Lempiainen, "Impact of the Base Station Antenna Beamwidth on Capacity in WCDMA Cellular Networks," in Proc. IEEE 57th Semiannual Vehicular Technology Conference, Apr. 2003, vol. 1, pp. 80-84.

[4] A. Wacker, J. Laiho-Steffens, K.Sipilä, K. Heiska, "The impact of the base station sectorisation on WCDMA radio network performance," in Proc. 50th IEEE Vehicular Technology Conference, 1999, pp. 26112615.

[5] D. Cabrera, J. Rodriguez, "Switched beam smart antenna BER performance analysis for 3G CDMA cellular communication", Computer Research Conference CRC2004, Puerto Rico, April 2004.

[6] A.U. Bhobe, P.L. Perini, "An overview of smart antenna technology for wireless communication", IEEE Aerospace Conference, Vol.2, pp. 875883, 2001.

[7] C.A. Balanis, “Antenna theory: Analysis and Design”, $3^{\text {rd }}$ ed, New York, Wiley, 2005.

[8] J. Hoydis, S. T. Brink, M. Debbah, "Massive MIMO in the UL/DL of Cellular Networks: How Many Antennas Do We Need?," Selected Areas in Communications, IEEE Journal on, vol.31, no.2, pp.160-171, February 2013.

[9] H. Huh, G. Caire, H.C. Papadopoulos, S.A. Ramprashad, "Achieving "Massive MIMO" Spectral Efficiency with a Not-so-Large Number of Antennas," Wireless Communications, IEEE Transactions on , vol.11, no.9, pp.3226-3239, September 2012

[10] H.W. Son, and N.H. Myung, "A deterministic ray tube method for microcellular wave propagation prediction model," Antennas and Propagation, IEEE Transactions, vol.47, no.8, pp.1344-1350, Aug 1999.

[11] D.N. Schettino, F.J.S. Moreira, C.G. Rego, "Efficient Ray Tracing for Radio Channel Characterization of Urban Scenarios," Magnetics, IEEE Transactions on , vol.43, no.4, pp.1305-1308, April 2007

[12] S. Soni, and A. Bhattacharya, "An efficient two-dimensional ray-tracing algorithm for modeling or urban microcellular environment", International Journal of Electronics and Communications (AEU), volume 66, issue 6, pp. 439-447, June 2012.

[13] F. Zhu, Q. Lin, J. Hu, "A directive patch antenna with a metamaterial cover," Microwave Conference Proceedings, 2005. APMC 2005. AsiaPacific Conference Proceedings, vol.3, no., pp. 3 pp., 4-7 Dec. 2005.

[14] H. Jun, Y. Chun-seng, L. Qing-chun, "A new patch antenna with meta material cover", Journal of Zhejiang University Science A, 2005. Doi:10.1631/jzus.2006.A0089

[15] J. S. Gomez-Diaz, J. Perruisseau-Carrier, "Microwave to THz properties of graphene and potential antenna applications," International Symposium on Antennas and Propagation (ISAP), 2012, pp.239-242, Oct. 29 2012-Nov. 22012

[16] I. Llatser, C. Kremers, D.N. Chigrin, J.M. Jornet, M.C. Lemme, A. Cabellos-Aparicio, and E. Alarcon, "Characterization of graphene-based nano-antennas in the terahertz band," 6th European Conference on Antennas and Propagation (EUCAP), 2012, pp.194-198, 26-30 March 2012. 